\title{
First countable spaces without point-countable $\pi$-bases
}

\author{
by \\ István Juhász, Lajos Soukup and Zoltán Szentmiklóssy (Budapest)
}

\begin{abstract}
We answer several questions of V. Tkachuk [Fund. Math. 186 (2005)] by showing that

(1) there is a ZFC example of a first countable, 0-dimensional Hausdorff space with no point-countable $\pi$-base (in fact, the minimum order of a $\pi$-base of the space can be made arbitrarily large);

(2) if there is a $\kappa$-Suslin line then there is a first countable GO-space of cardinality $\kappa^{+}$in which the order of any $\pi$-base is at least $\kappa$;

(3) it is consistent to have a first countable, hereditarily Lindelöf regular space having uncountable $\pi$-weight and $\omega_{1}$ as a caliber (of course, such a space cannot have a point-countable $\pi$-base).
\end{abstract}

1. Introduction. V. Tkachuk in [9] has recently proved under $\mathrm{CH}$ that any first countable Hausdorff space that is Lindelöf or CCC has a pointcountable $\pi$-base. (Actually, in [9] all spaces are assumed to be Tikhonov, but the proof only needs Hausdorff.) Tkachuk's motivation was to extend (at least partially) Shapirovskiı̌'s celebrated ZFC result saying that any countably tight compactum has a point-countable $\pi$-base, from compact spaces to Lindelöf ones. So it was natural to ask if his use of $\mathrm{CH}$ was necessary. Also, 27 years after Shapirovski1's result was published, Tkachuk could not come up with even a consistent example of a first countable space without a point-countable $\pi$-base.

Our aim here is to remedy this situation and provide ZFC (and several consistent) examples of first countable (Tikhonov) spaces without pointcountable $\pi$-bases, as well as examples which show that Tkachuk's $\mathrm{CH}$ results cannot be proved in ZFC alone. In this manner we succeeded in answering seven of the twelve questions that were listed at the end of [9].

2000 Mathematics Subject Classification: 54A25, 54A35, 54D20, 54D70, 54F05.

Key words and phrases: first countable space, point-countable $\pi$-base, Lindelöf space, CCC space, caliber.

Research supported by OTKA grant no. 61600 . 
In what follows, we shall use the notation and terminology of [4]. In particular, $\pi s w(X)$ denotes the $\pi$-separating weight of $X$, that is, the minimum order of a $\pi$-base of the space $X$ (see p. 74 of [4]). Note that $\pi s w(X) \leq \omega$ is then equivalent to the statement: $X$ has a point-countable $\pi$-base.

2. ZFC examples. The key to Tkachuk's above mentioned $\mathrm{CH}$ results in [9] was his Theorem 3.1 which says that if $X$ has countable tightness and $\pi$-character, and moreover $d(X) \leq \omega_{1}$, then $\pi s w(X) \leq \omega$. In his list of problems (Problem 4.11), Tkachuk asked if the assumption of countable tightness could be omitted here. It is immediate from our next result that this question has an affirmative answer.

Theorem 1. Let $X$ be any topological space with $d(X) \leq \pi \chi(X)^{+}$. Then $\pi s w(X) \leq \pi \chi(X)$.

Proof. Let us set $\pi \chi(X)=\kappa$. If $d(X) \leq \kappa$ then we even have $\pi(X)=\kappa$. So we may assume $d(X)=\kappa^{+}$and, as is well-known, we may then fix a dense set $D=\left\{x_{\alpha}: \alpha<\kappa^{+}\right\}$that is left-separated in this well-ordering. This means that for every $\alpha<\kappa^{+}$there is a neighbourhood $U_{\alpha}$ of $x_{\alpha}$ with

$$
\left\{x_{\beta}: \beta<\alpha\right\} \cap U_{\alpha}=\emptyset .
$$

Let us now fix a local $\pi$-base $\mathcal{B}_{\alpha}$ of the point $x_{\alpha}$ such that $\left|\mathcal{B}_{\alpha}\right| \leq \kappa$ and $B \subset U_{\alpha}$ whenever $B \in \mathcal{B}_{\alpha}$. Then $\mathcal{B}=\bigcup\left\{\mathcal{B}_{\alpha}: \alpha<\kappa^{+}\right\}$is a $\pi$-base of $X$ such that for every $x_{\beta} \in D$ we have

$$
\operatorname{ord}\left(x_{\beta}, \mathcal{B}\right)=\left|\left\{B \in \mathcal{B}: x_{\beta} \in B\right\}\right| \leq \kappa .
$$

We claim that we then have

$$
\operatorname{ord}(\mathcal{B})=\sup \{\operatorname{ord}(x, \mathcal{B}): x \in X\} \leq \kappa
$$

as well. Assume, on the contrary, that $\operatorname{ord}(x, \mathcal{B})=\kappa^{+}$for some $x \in X$. Since $\pi \chi(x, X) \leq \kappa$, this implies that there are $\kappa^{+}$members of $\mathcal{B}$ (containing $x$ ) that include a fixed non-empty open set $V$. This, however, is impossible because $D \cap V \neq \emptyset$.

We may now turn to our first aim, which is to produce, in ZFC, first countable spaces without point-countable $\pi$-bases.

TheOREM 2. There is a first countable, 0-dimensional Hausdorff (hence Tikhonov) space $X$ with $\pi s w(X) \geq \aleph_{\omega}$.

Proof. The underlying set of our space is $X=\prod\left\{\omega_{n}: n<\omega\right\}$. For $f, g \in X$ we write $f \leq g$ to denote that $f(n) \leq g(n)$ for all $n<\omega$. The topology $\tau$ that we shall consider on $X$ will be generated by all sets of the form $U_{n}(f)$ (with $f \in X$ and $n<\omega$ ), where

$$
U_{n}(f)=\{g \in X: f \leq g \text { and } f\lceil n=g\lceil n\} .
$$


Note that if $g \in U_{n}(f)$ then $U_{n}(g) \subset U_{n}(f)$, and if $g \notin U_{n}(f)$ then there is $k<\omega$ such that $U_{k}(g) \cap U_{n}(f)=\emptyset$. It follows that, for any $f \in X$, the family $\left\{U_{n}(f): n<\omega\right\}$ forms a clopen neighbourhood base of $f$ with respect to the topology $\tau$, and consequently the space $\langle X, \tau\rangle$ is indeed first countable, 0-dimensional, and Hausdorff.

It is also easy to see from the definitions that if $\left\{U_{n_{\alpha}}\left(f_{\alpha}\right): \alpha<\kappa\right\}$ is a $\pi$-base of $\tau$ then $\left\{f_{\alpha}: \alpha<\kappa\right\}$ must be cofinal in the partial order $\langle X, \leq\rangle$. But it is well-known that the cofinality of $\langle X, \leq\rangle$ is greater than $\aleph_{\omega}$, so $\pi(X)>\aleph_{\omega}$. (Actually, it is easy to see that $\pi(X)=\operatorname{cf}(\langle X, \leq\rangle)$ but we shall not need this.)

Next we claim that, for any $k<\omega$, the pair $\left(\aleph_{\omega+1}, \aleph_{k}\right)$ is a pair caliber of the space $X$, i.e. among any $\aleph_{\omega+1}$ open sets one can find $\aleph_{k}$ whose intersection is non-empty. Without any loss of generality, it suffices to check this for a family of basic open sets of the form $\left\{U_{n}(f): f \in F\right\}$ where $F \in[X]^{\aleph_{\omega+1}}$ and $n>k$ is fixed. We may also assume that $f\lceil n=\sigma$ for a fixed $\sigma \in \prod_{i<n} \omega_{i}$ whenever $f \in F$. Now let $G \subset F$ with $|G|=\aleph_{k}$. Then there is $g \in X$ with $g\lceil n=\sigma$ and $f(i)<g(i)$ for all $i \geq n$ and $f \in G$. But then we have $g \in \bigcap\left\{U_{n}(f): f \in G\right\}$.

Putting together the previous two paragraphs we conclude that the order of any $\pi$-base of $\langle X, \tau\rangle$ must be at least $\aleph_{\omega}$, that is, $\pi s w(X) \geq \aleph_{\omega}$.

It is clear that if we replace in the above proof the sequence $\left\langle\omega_{n}: n<\omega\right\rangle$ with any other strictly increasing $\omega$-sequence of regular cardinals, say $\left\langle\kappa_{n}\right.$ : $n<\omega\rangle$, then we obtain a first countable, 0 -dimensional space in which the order of any $\pi$-base is at least $\sum_{n<\omega} \kappa_{n}$.

The referee has pointed out that the method of constructing such spaces was published by Todorčević in [10, Theorem 0.5] (of course, the fact that they do not have a point-countable $\pi$-base is not mentioned there).

The cardinality of our example is $\aleph_{\omega}^{\aleph_{0}}$, which is much larger than the optimal value $\aleph_{2}$ permitted by Theorem 1 . So it is natural to raise the question if we could find other examples of smaller cardinality. It turns out that we can do slightly better by choosing an appropriate subspace $Y$ of the space $X$ from Theorem 2. First, however, we need to fix some notation. For $f, g \in X=\prod\left\{\omega_{n}: n<\omega\right\}$ we write $f<{ }^{*} g$ to denote that $|\{n<\omega: f(n) \geq g(n)\}|$ is finite, i.e. $f$ is below $g$ modulo finite. Similarly, we write $f={ }^{*} g$ to denote that $|\{n<\omega: f(n) \neq g(n)\}|$ is finite. Finally, it is well-known that there is in $X$ a transfinite sequence of order type $\omega_{\omega+1}$ that is increasing with respect to $<^{*}$.

TheOREM 3. Let $\left\{f_{\alpha}: \alpha<\omega_{\omega+1}\right\} \subset X$ be an increasing sequence with respect to $<^{*}$ and set

$$
Y=\left\{f \in X: \exists \alpha<\omega_{\omega+1} \text { with } f={ }^{*} f_{\alpha}\right\} .
$$


Then the subspace $Y$ of $X$, with the subspace topology inherited from $\tau$, also satisfies $\pi s w(Y) \geq \aleph_{\omega}$.

Proof. The proof is very similar to that of Theorem 2. First we note that, trivially, we again have $\pi(Y)>\aleph_{\omega}$. Next, $\left(\aleph_{\omega+1}, \aleph_{k}\right)$ is a pair caliber of $Y$ for each $k<\omega$. To see this, we again consider a family $\left\{U_{n}(f): f \in F\right\}$ where $F \in[Y]^{\aleph_{\omega+1}}$ and $n>k>0$, and also $f\left\lceil n=\sigma\right.$ for a fixed $\sigma \in \prod_{i<n} \omega_{i}$ whenever $f \in F$. Let us choose any subset $G \subset F$ with $|G|=\aleph_{k}$. Then there is an ordinal $\alpha<\omega_{\omega+1}$ such that $g<^{*} f_{\alpha}$ for all $g \in G$. We may find an integer $m \geq n$ such that the set

$$
G^{*}=\left\{g \in G: \forall i \geq m\left(g(i)<f_{\alpha}(i)\right)\right\}
$$

also has cardinality $\aleph_{k}$.

Note that if $n \leq j<m$ then $\left\{g(j): g \in G^{*}\right\}$ is bounded in $\omega_{j}$, hence we may find a function $f \in Y$ such that $f\lceil n=\sigma$, if $n \leq j<m$ then $g(j)<f(j)$ for all $g \in G^{*}$, and $f(i)=f_{\alpha}(i)$ whenever $m \leq i<\omega$. Clearly, we then have $f \in \bigcap\left\{U_{n}(g): g \in G^{*}\right\} \cap Y$.

We have been unable to produce a ZFC example of a first countable space without a point-countable $\pi$-base of cardinality less than $\aleph_{\omega+1}$. This leads us to the following intriguing open question.

Problem 4. Is there, in ZFC, a first countable (Tikhonov) space of cardinality less than $\aleph_{\omega}$ that has no point-countable $\pi$-base?

Actually, at present we do not even have such an example of cardinality $\aleph_{\omega}$. We conjecture, however, that having such an example is equivalent to having one of size $<\aleph_{\omega}$. In fact, we can confirm this conjecture under the assumption $2^{\aleph_{1}}<\aleph_{\omega}$.

Theorem 5. Assume that $2^{\aleph_{1}}<\aleph_{\omega}$ and $X$ is a first countable space of cardinality $\aleph_{\omega}$. If every subspace of $X$ of cardinality $<\aleph_{\omega}$ has a pointcountable $\pi$-base then so does $X$.

Proof. Let us start by giving a (very natural) definition. A family $\mathcal{B}$ of non-empty open sets in $X$ is said to be an outer $\pi$-base of a subspace $Y \subset X$ if for every open set $U$ with $U \cap Y \neq \emptyset$ there is a member $B \in \mathcal{B}$ such that $B \subset U$. We claim that, under the assumptions of our theorem, every subspace of $X$ of cardinality $<\aleph_{\omega}$ has a point-countable outer $\pi$-base. Thus if $X=\bigcup_{n<\omega} Y_{n}$ where $\left|Y_{n}\right|<\aleph_{\omega}$ for all $n<\omega$ and $\mathcal{B}_{n}$ is a point-countable outer $\pi$-base of $Y_{n}$ in $X$ then $\bigcup_{n<\omega} \mathcal{B}_{n}$ is a point-countable $\pi$-base of $X$.

To prove the above claim let us consider an $\omega_{1}$-closed elementary submodel $M$ of a "universe" $H(\theta)$ with $|M|<\aleph_{\omega}$. (As usual, here $\theta$ is a large enough regular cardinal, $H(\theta)$ is the collection of all sets of hereditary cardinality $<\theta$, and for $M$ to be $\omega_{1}$-closed means that $[M]^{\leq \omega_{1}} \subset M$.) The regular cardinal $\theta$ is chosen so large that $H(\theta)$ (and also $M$ ) contains $X$ and 
everything else that is relevant, e.g. a map $\mathcal{V}$ that assigns to every point $x \in X$ a countable open neighbourhood base $\mathcal{V}_{x}$. Now, $2^{\aleph_{1}}<\aleph_{\omega}$ implies that for every $Y \in[X]^{<\aleph_{\omega}}$ there is such an elementary submodel $M$ with $Y \subset M$. Consequently, our claim will be proven if we show that $X \cap M$ has a point-countable outer $\pi$-base in $X$ whenever $M$ is as above.

To see this, note first that for every $x \in X \cap M$ we have $\mathcal{V}_{x} \in M$ and hence $\mathcal{V}_{x} \subset M$ as well. Consequently, $\mathcal{V}_{M}=\bigcup\left\{\mathcal{V}_{x}: x \in X \cap M\right\} \subset M$ is an outer base of $X \cap M$ in $X$, hence we may choose a subfamily $\mathcal{B} \subset \mathcal{V}_{M}$ such that $\mathcal{B}\lceil M=\{B \cap M: B \in \mathcal{B}\}$ is a point-countable $\pi$-base of the subspace $X \cap M$.

It suffices to show now that $\mathcal{B}$ is a point-countable outer $\pi$-base of $X \cap M$ in $X$. Indeed, $\mathcal{B}$ is point-countable, for if $\mathcal{U} \in[\mathcal{B}]^{\omega_{1}}$ then $\mathcal{U} \in M$ because $M$ is $\omega_{1}$-closed, and thus $\bigcap \mathcal{U} \neq \emptyset$ would imply $\bigcap \mathcal{U} \cap M \neq \emptyset$, contradicting the fact that $\mathcal{B} \backslash M$ is point-countable. (Here we used the fact that, by elementarity, the correspondence $B \mapsto B \cap M$ is one-to-one on $\mathcal{B} \subset M$.) Finally, $\mathcal{B}$ is an outer $\pi$-base of $X \cap M$ in $X$, because if $U$ is open with $x \in U \cap M \neq \emptyset$ then there is $V \in \mathcal{V}_{x} \subset M$ with $V \subset U$, hence if $B \in \mathcal{B}$ with $B \cap M \subset V \in M$ then we also have $B \subset V \subset U$.

3. Examples from higher Suslin lines. We start this section by giving a theorem that, quite naturally, will turn out to be very useful in finding (first countable) spaces without point-countable $\pi$-bases.

TheOrem 6. Assume that $X$ is a topological space which has a $\pi$-base $\mathcal{B}$ such that $\operatorname{ord}(\mathcal{B})^{+}<d(X)$. Then $X$ has a discrete subspace $D$ with $|D| \geq$ $d(X)$.

Proof. Let us first choose a point $x_{B} \in B$ from each $B \in \mathcal{B}$. Then the set $S=\left\{x_{B}: B \in \mathcal{B}\right\}$ is dense in $X$, hence we have

$$
|S| \geq d(X)>\operatorname{ord}(\mathcal{B})^{+} \text {. }
$$

We now define a set mapping $F$ on $S$ by setting, for any $x \in S$,

$$
F(x)=\left\{x_{B} \in S: x \in B\right\} \in[S] \leq \operatorname{ord}(\mathcal{B}) .
$$

By Hajnal's set mapping theorem (see [2]) there is then a free set $D \subset S$ for the set mapping $F$ with $|D|=|S|$. This means that for every $x \in D$ we have $D \cap F(x) \subset\{x\}$. But every member of $D$ is of the form $x_{B}$ for some $B \in \mathcal{B}$, and we claim that for this point we have $B \cap D=\left\{x_{B}\right\}$. Indeed, $x_{B} \in B \cap D$ is obvious, and if $x \in D$ is different from $x_{B}$ then $x_{B} \notin F(x)$ implies $x \notin B$. Consequently, $D$ is as required.

The referee has pointed out that Theorem 6 is an easy consequence of the following result of Shapirovskiu ([7, Lemma 3.1], see also $[4,3.26])$ : If $\mathcal{B}$ is any family of non-empty open sets in a space $X$ with $\operatorname{ord}(\mathcal{B}) \leq \kappa$ then there are discrete subspaces $\left\{D_{\alpha}: \alpha<\kappa^{+}\right\}$of $X$ such that $\bigcup\left\{D_{\alpha}: \alpha<\kappa^{+}\right\} \cap B \neq \emptyset$ 
for any $B \in \mathcal{B}$. Since our proof of Theorem 6 is quite different and very short, for the reader's convenience we decided to keep it.

It is an immediate consequence of Theorem 6 that a space $X$ satisfying $s(X)<d(X) \geq \omega_{2}$ cannot have a point-countable $\pi$-base. Unfortunately, we do not know if there is in ZFC a first countable Tikhonov space like that. (Recall that solving Tkachuk's problems from [9] requires Tikhonov examples.) If, however, we are satisfied with Hausdorff examples then we are much better off. In fact, it was shown in [3] that there is a natural left-separated refinement $\sigma$ of the euclidean topology $\tau$ on the real line $\mathbb{R}$ that is first countable and hereditarily Lindelöf. Consequently, by Theorem 6 , a subspace of $\langle\mathbb{R}, \sigma\rangle$ which is left-separated in order-type $\omega_{2}$, and thus of density $\omega_{2}$, has no point-countable $\pi$-base. This shows that, at least for Hausdorff spaces, Tkachuk's $\mathrm{CH}$ results mentioned in the introduction simply fail to hold without $\mathrm{CH}$, for $\langle\mathbb{R}, \sigma\rangle$ is hereditarily Lindelöf. Actually, it is very easy to show that something stronger than CCC can be established for such a subspace, namely that $\omega_{1}$ is a caliber of it. For Hausdorff spaces, this settles one more question of Tkachuk from [9]. In the next section we shall produce (consistent) Tikhonov examples with these properties but that will require more work.

Next we shall consider higher Suslin lines; these are ordered spaces whose spread (equal in this case to cellularity) is less than their density. More precisely, we shall consider first countable variations of them that retain this property. For different purposes, this construction was already used in Theorem 1.1 of [5], although there $\mathrm{CH}$ was additionally assumed.

Let $\kappa$ be an infinite cardinal. We shall call a continuous linear order $\langle L,<\rangle$, equipped with the order topology generated by $<$, a $\kappa$-Suslin line if there are no more than $\kappa$ disjoint open intervals in $L$ (i.e. $c(L) \leq \kappa$ ), although the density $d(L)$ of $L$ is larger than $\kappa$. (It is known that the existence of a $\kappa$-Suslin line is equivalent to the existence of a $\kappa$-Suslin tree, but this will be irrelevant for us.) Thus, an ordinary Suslin line is the same as an $\omega$-Suslin line and by a higher Suslin line we mean a $\kappa$-Suslin line where $\kappa>\omega$.

The main result of this section is the following theorem, which in particular yields a consistent example of a first countable GO-space without a point-countable $\pi$-base of the minimum possible cardinality $\omega_{2}$. (Recall that GO-spaces, or generalized ordered spaces, are the subspaces of linearly ordered spaces.)

THEOREM 7. If there is a $\kappa$-Suslin line $\langle L,<\rangle$ then there is a first countable GO-space $X$ with $|X|=\kappa^{+}$and $\pi s w(X)=\kappa$.

Proof. Let $Z$ be the set of all those points $x \in L$ that have left character $\omega$, that is, the open half line $(\leftarrow, x)$ has cofinality $\omega$ with respect to $<$. Since $\langle L,<\rangle$ is continuous, $Z$ is dense in $L$. It follows that $d(Z)=d(L)=\kappa^{+}$ 
because $d(L) \leq c(L)^{+}$for any linearly ordered space $L$ (see e.g. [1]). Now let $X$ be any dense subspace of $Z$ (and hence of $L$ ) with $|X|=\kappa^{+}$.

We consider $X$ with the left Sorgenfrey topology $\sigma$, i.e. for any $x \in X$ the half-open intervals $(y, x]$ form a $\sigma$-local base. Then $\sigma$ is finer than the order topology on $X$, hence the density of $\langle X, \sigma\rangle$ must be larger than $\kappa$. It is clear from the definition that $\sigma$ is a first countable topology.

Also, $\langle X, \sigma\rangle$ is a GO-space because it is homeomorphic to the subspace topology on $X \times\{0\}$ inherited from the order topology on $L \times 2$ taken with the lexicographic order. Finally, we have $c(X, \sigma)=c(L)$; moreover, $s(X, \sigma)=c(X, \sigma)$ is known to hold for GO-spaces (see 2.23 of [4]). Consequently, $s(X) \leq \kappa<d(X)$ and so Theorem 6 implies $\pi s w(X) \geq \kappa$. By Theorem 1, then $\pi s w(X)=\kappa$.

In particular, the existence of an $\omega_{1}$-Suslin line implies the existence of a first countable GO-space of cardinality $\omega_{2}$ without a point-countable $\pi$-base.

Finally, we mention here the curious fact that it is an outstanding open question of set theory whether one can find a model of ZFC that does not contain any higher Suslin line. Consequently, there is a chance that Theorem 7 yields a ZFC example of a first countable GO-space with no pointcountable $\pi$-base.

4. Examples from subfamilies of $\mathcal{P}(\omega)$. In this section we are going to introduce a (quite simple but apparently new) way of constructing first countable, 0-dimensional Hausdorff topologies on subfamilies of $\mathcal{P}(\omega)$, the power set of $\omega$. Then we shall use some of the spaces obtained in this manner to present examples that demonstrate the necessity of the use of $\mathrm{CH}$ in Tkachuk's results mentioned in the introduction.

We start by fixing some notation and terminology. We shall use $\mathcal{U}$ to denote the family of all co-finite subsets of $\omega$. For a given family $\mathcal{I} \subset \mathcal{P}(\omega)$ and for $I \in \mathcal{I}$ and $U \in \mathcal{U}$ we put

$$
[I, U)_{\mathcal{I}}=\{J \in \mathcal{I}: I \subset J \subset U\} .
$$

If $\mathcal{I}=\mathcal{P}(\omega)$ then we shall omit the subscript.

Finally, we say that the family $\mathcal{I} \subset \mathcal{P}(\omega)$ is stable if $I \in \mathcal{I}$ and $I={ }^{*} J$ for $J \subset \omega$ imply $J \in \mathcal{I}$ as well. (Of course, here $I={ }^{*} J$ means that $I$ and $J$ are equal mod finite, i.e. their symmetric difference $I \triangle J$ is finite.)

Definition 8. Let us fix a family $\mathcal{I} \subset \mathcal{P}(\omega)$. We shall denote by $\tau_{\mathcal{I}}$ the topology on $\mathcal{I}$ generated by all sets of the form $[I, U)_{\mathcal{I}}$, where $I \in \mathcal{I}$ and $U \in \mathcal{U}$, and by $X_{\mathcal{I}}$ the space $\left\langle\mathcal{I}, \tau_{\mathcal{I}}\right\rangle$.

Of course, $X_{\mathcal{I}}$ is identical with the appropriate subspace of the maximal such space $X_{\mathcal{P}(\omega)}$. A few basic (pleasant) properties of the spaces $X_{\mathcal{I}}$ are given in the following proposition. 
Proposition 9. The spaces $X_{\mathcal{I}}$ are first countable, 0-dimensional and Hausdorff.

Proof. It suffices to show this for $\mathcal{I}=\mathcal{P}(\omega)$ because all three properties are inherited by subspaces.

Observe first that if $J \in[I, U) \cap\left[I^{\prime}, U^{\prime}\right)$ then

$$
J \in\left[J, U \cap U^{\prime}\right) \subset[I, U) \cap\left[I^{\prime}, U^{\prime}\right),
$$

hence the "intervals" $[I, U)$ form an open basis of $\tau_{\mathcal{P}(\omega)}$, and $\{[I, U): I \subset$ $U \in \mathcal{U}\}$ forms a countable neighbourhood base of the point $I$ of $X_{\mathcal{I}}$.

Next, if $J \notin[I, U)$ then either $J \backslash U \neq \emptyset$ and then $[J, \omega) \cap[I, U)=\emptyset$, or $J \subset U$ and $I \backslash J \neq \emptyset$. In the latter case we may pick $n \in I \backslash J$ and have $J \subset U \backslash\{n\}$, and also $[J, U \backslash\{n\}) \cap[I, U)=\emptyset$ because $n \in I$. This means that all basic open sets $[I, U)$ are also closed, hence $X_{\mathcal{P}(\omega)}$ is indeed 0-dimensional.

Finally, for every $I \in \mathcal{P}(\omega)$ we have

$$
\bigcap\{[I, U): I \subset U \in \mathcal{U}\}=\{I\}
$$

implying that $X_{\mathcal{P}(\omega)}$ is also Hausdorff.

For any family $\mathcal{I} \subset \mathcal{P}(\omega)$ we shall denote by $\operatorname{cof}(\mathcal{I})$ the cofinality of the partial order $\langle\mathcal{I}, \subset\rangle$. Also, we say that a cardinal number $\kappa$ is a set caliber of $\mathcal{I}$ if for every subfamily $\mathcal{J} \in[\mathcal{I}]^{\kappa}$ there are $\mathcal{K} \in[\mathcal{J}]^{\kappa}$ and $I \in \mathcal{I}$ such that $\bigcup \mathcal{K} \subset I$ or, less formally, among any $\kappa$ members of $\mathcal{I}$ there are $\kappa$ that have an upper bound in $\mathcal{I}$. We now connect these concepts concerning $\mathcal{I}$ with the properties of the associated space $X_{\mathcal{I}}$.

Proposition 10. For any subfamily $\mathcal{I} \subset \mathcal{P}(\omega)$ we have

(i) $d\left(X_{\mathcal{I}}\right)=\operatorname{cof}(\mathcal{I}) \cdot \omega$;

(ii) if $\mathcal{I}$ is stable and $\kappa$ is a cardinal with $\operatorname{cf}(\kappa)>\omega$ then $\kappa$ is a caliber of the space $X_{\mathcal{I}}$ if and only if $\kappa$ is a set caliber of $\mathcal{I}$.

Proof. (i) and the left-to-right direction of (ii) follow immediately from the fact that $\mathcal{K} \subset \mathcal{I}$ has an upper bound in $\mathcal{I}$ iff $\bigcap\left\{[I, \omega)_{\mathcal{I}}: I \in \mathcal{K}\right\} \neq \emptyset$. To see the other direction, assume that $\kappa$ is a set caliber of $\mathcal{I}$ and consider a family $\mathcal{B}$ of $\kappa$ basic open sets. Since $\operatorname{cf}(\kappa)>\omega$ we may assume that $\mathcal{B}=\{[I, U): I \in \mathcal{J}\}$ for $\mathcal{J} \in[\mathcal{I}]^{\kappa}$ and a fixed $U \in \mathcal{U}$. By our assumption there is a $\mathcal{K} \in[\mathcal{J}]^{\kappa}$ which has an upper bound $K \in \mathcal{I}$. Then $K \cap U \in \mathcal{I}$, as $\mathcal{I}$ is stable and

$$
K \cap U \in \bigcap\{[I, U): I \in \mathcal{K}\}
$$

After these preparatory propositions we can now present a result that will yield further nice examples of first countable spaces without point-countable $\pi$-bases. 
TheOREM 11. Assume that $\mathcal{I} \subset \mathcal{P}(\omega)$ is stable, $\operatorname{cof}(\mathcal{I})>\omega$, and $\omega_{1}$ is a set caliber of $\mathcal{I}$. Then $\pi s w\left(X_{\mathcal{I}}\right)>\omega$.

Proof. Since $X_{\mathcal{I}}$ is first countable, and by Proposition 10(i), we have

$$
\pi\left(X_{\mathcal{I}}\right)=d\left(X_{\mathcal{I}}\right)=\operatorname{cof}(\mathcal{I})>\omega
$$

But, in view of Proposition 10(ii), $\omega_{1}$ is a caliber of $X_{\mathcal{I}}$, and hence no $\pi$-base of $X_{\mathcal{I}}$ can be point-countable.

COROLlary 12. Assume that there is a mod finite strictly increasing $\omega_{2}$-sequence in $\mathcal{P}(\omega)$. Then there is a first countable, 0-dimensional and Hausdorff space of cardinality $\omega_{2}$ which has $\omega_{1}$ as a caliber. In particular, $\mathrm{MA}_{\omega_{1}}$ implies the existence of such a space.

Proof. Let $\left\{A_{\alpha}: \alpha<\omega_{2}\right\} \subset \mathcal{P}(\omega)$ be a mod finite strictly increasing $\omega_{2}$-sequence, i.e. $\left|A_{\alpha} \backslash A_{\beta}\right|<\omega$ and $\left|A_{\beta} \backslash A_{\alpha}\right|=\omega$ whenever $\alpha<\beta<\omega_{2}$. It is obvious that the family

$$
\mathcal{I}=\left\{I \subset \omega: \exists \alpha<\omega_{2} \text { with } I={ }^{*} A_{\alpha}\right\}
$$

is stable and satisfies $|\mathcal{I}|=\operatorname{cof}(\mathcal{I})=\omega_{2}$. Next, we claim that $\omega_{1}$ is a set caliber of $\mathcal{I}$.

To see this, consider any family $\mathcal{J}=\left\{I_{\alpha}: \alpha \in a\right\} \subset \mathcal{I}$ where $a \in\left[\omega_{2}\right]^{\omega_{1}}$ and $I_{\alpha}=^{*} A_{\alpha}$ for all $\alpha \in a$, and pick $\beta<\omega_{2}$ such that $a \subset \beta$. Then $\left|A_{\alpha} \backslash A_{\beta}\right|<\omega$ for all $\alpha \in a$, hence there is a fixed $s \in[\omega]^{<\omega}$ such that

$$
b=\left\{\alpha \in a: A_{\alpha} \backslash A_{\beta} \subset s\right\}
$$

is uncountable, while $s \cup A_{\beta}$ is an upper bound of $\left\{I_{\alpha}: \alpha \in b\right\}$ in $\mathcal{I}$. By Theorem 11, the space $X_{\mathcal{I}}$ is as required.

This result takes care of Problems 4.6 and 4.7 from [9] by showing that it is consistent to have first countable Tikhonov spaces with caliber $\omega_{1}$ (and hence also CCC) without any point-countable $\pi$-base. With some further elaboration we shall find examples that, in addition, are also hereditarily Lindelöf, and thus provide a solution to Problem 4.3 from [9] as well.

Theorem 13. Let $\left\{A_{\alpha}: \alpha<\omega_{2}\right\} \subset \mathcal{P}(\omega)$ be a mod finite strictly increasing $\omega_{2}$-sequence with the additional property that in every uncountable index set $a \in\left[\omega_{2}\right]^{\omega_{1}}$ there is a pair $\{\alpha, \beta\} \in[a]^{2}$ such that $A_{\alpha} \subset A_{\beta}$ (i.e. $A_{\alpha}$ is really a subset of $A_{\beta}$, not just mod finite). Then, with $\mathcal{I}$ defined as in Corollary 12 , the space $X_{\mathcal{I}}$ is hereditarily Lindelöf.

Proof. Assume, on the contrary, that $X_{\mathcal{I}}$ has an uncountable rightseparated subspace. Without loss of generality this may be taken of the form $\left\{I_{\alpha}: \alpha \in a\right\}$, right-separated in the natural well-ordering of its indices, where $a \in\left[\omega_{2}\right]^{\omega_{1}}$ and $I_{\alpha}={ }^{*} A_{\alpha}$ for all $\alpha \in a$. Moreover, we may assume that we have a fixed $U \in \mathcal{U}$ such that $\left[I_{\alpha}, U\right)_{\mathcal{I}}$ is a right-separating neighbourhood of $I_{\alpha}$ for any $\alpha \in a$. 
Now, there is a fixed finite set $s \in[\omega]^{<\omega}$ such that

$$
b=\left\{\alpha \in a: I_{\alpha} \triangle A_{\alpha}=s\right\}
$$

is uncountable. By our assumption, there is a pair $\{\alpha, \beta\} \in[b]^{2}$ (with $\alpha<\beta)$ for which $A_{\alpha} \subset A_{\beta}$ and hence $I_{\alpha} \subset I_{\beta}$. This, however, would imply $I_{\beta} \in\left[I_{\alpha}, U\right)_{\mathcal{I}}$, contradicting the fact that $\left[I_{\alpha}, U\right)_{\mathcal{I}}$ is a right-separating neighbourhood of $I_{\alpha}$.

Note that a space as in Theorem 13 is a first countable L-space, hence unlike the spaces in Corollary 12, it does not exist under $\mathrm{MA}_{\omega_{1}}$ (see [8]). Instead, there is a "natural" forcing construction that produces mod finite strictly increasing $\omega_{2}$-sequences in $\mathcal{P}(\omega)$ with the additional property required in Theorem 13.

THEOREM 14. There is a CCC forcing that, to any ground model, adds a mod finite strictly increasing sequence $\left\{A_{\alpha}: \alpha<\omega_{2}\right\} \subset \mathcal{P}(\omega)$ in any uncountable subsequence of which there are two members with proper inclusion.

Proof. Let $\mathbb{P}$ consist of those finite functions $p \in \mathrm{Fn}\left(\omega_{2} \times \omega, 2\right)$ for which $\operatorname{dom}(p)=a \times n$ with $a \in\left[\omega_{2}\right]^{<\omega}$ and $n<\omega$. We define $p^{\prime} \leq p$ (i.e. $p^{\prime}$ extends $p)$ as follows: $p^{\prime} \supset p$, and $p^{\prime}(\alpha, i)=1$ implies $p^{\prime}(\beta, i)=1$ whenever $\alpha, \beta \in a$ with $\alpha<\beta$ and $i \in n^{\prime} \backslash n$ (of course, here $\operatorname{dom}(p)=a \times n$ and $\operatorname{dom}\left(p^{\prime}\right)=$ $\left.a^{\prime} \times n^{\prime}\right)$. It is straightforward to show that $\langle\mathbb{P}, \leq\rangle$ is a CCC notion of forcing.

Let $G \subset \mathbb{P}$ be generic. Then it follows from standard density arguments that $g=\cup G$ maps $\omega_{2} \times \omega$ into 2 and if we set

$$
A_{\alpha}=\{i<\omega: g(\alpha, i)=1\}
$$

then $\left\{A_{\alpha}: \alpha<\omega_{2}\right\}$ is mod finite strictly increasing.

To finish the proof, assume that $p \in \mathbb{P}$ forces that $\dot{h}$ is an order preserving injection of $\omega_{1}$ into $\omega_{2}$. It suffices to show that $p$ has an extension $q$ which forces $A_{\dot{h}(\xi)} \subset A_{\dot{h}(\eta)}$ for some $\xi<\eta<\omega_{1}$.

To see this, choose first for each $\xi<\omega_{1}$ a condition $p_{\xi} \leq p$ and an ordinal $\alpha_{\xi}<\omega_{2}$ such that $p_{\xi} \Vdash \dot{h}(\xi)=\alpha_{\xi}$. We may assume without any loss of generality that for some $n<\omega$ we have $\operatorname{dom}\left(p_{\xi}\right)=a_{\xi} \times n$ and $\alpha_{\xi} \in a_{\xi}$ for all $\xi$. Using standard $\triangle$-system and counting arguments, it is easy to find then $\xi<\eta<\omega_{1}$ such that $p_{\xi}$ and $p_{\eta}$ are compatible as functions and for any $i<n$ we have $p_{\xi}\left(\alpha_{\xi}, i\right)=p_{\eta}\left(\alpha_{\eta}, i\right)$. But then we have $q=p_{\xi} \cup p_{\eta} \in \mathbb{P}$ and $q \leq p$; moreover, it is obvious that $q$ forces $A_{\alpha_{\xi}} \subset A_{\alpha_{\eta}}$ and hence $A_{\dot{h}(\xi)} \subset A_{\dot{h}(\eta)}$ as well.

From Theorems 13 and 14 we immediately obtain a joint solution to Problems 4.3 and 4.7 (and hence 4.6) of Tkachuk from [9].

COROLlary 15. It is consistent that there exists a first countable, hereditarily Lindelöf 0-dimensional space $X$ of size $\omega_{2}$ which has no point-countable $\pi$-base while $\omega_{1}$ is a caliber of $X$. 
Let us recall here that the failure of $\mathrm{CH}$ is not sufficient to produce a mod finite strictly increasing $\omega_{2}$-sequence in $\mathcal{P}(\omega)$, the basic ingredient of our examples in this section. In fact, Kunen proved (see e.g. [6]) that if one adds $\omega_{2}$ Cohen reals to a model of $\mathrm{CH}$ then no such sequence exists in the extension. Actually, we have shown the following strengthening of this: In the same model, if $\omega_{1}$ is a set caliber of a subfamily $\mathcal{I}$ of $\mathcal{P}(\omega)$ then $\operatorname{cof}(\mathcal{I}) \leq \omega$. This implies that we may not use the methods of this section to find similar examples just assuming the negation of $\mathrm{CH}$. The following natural problem can thus be raised.

Problem 16. Does $2^{\omega}>\omega_{1}$ imply the existence of a first countable Lindelöf and/or CCC Tikhonov space having no point-countable $\pi$-base?

\section{References}

[1] H. R. Bennett and and T. G. McLaughlin, A Selective Survey of Axiom-Sensitive Results in General Topology, Texas Tech Univ. Math. Ser. 12, Lubbock, TX, 1976.

[2] A. Hajnal, Proof of a conjecture of S. Ruziewicz, Fund. Math. 50 (1961/1962), $123-128$.

[3] A. Hajnal and I. Juhász, On hereditarily $\alpha$-Lindelöf and hereditarily $\alpha$-separable spaces, Ann. Univ. Sci. Budapest Sect. Math. 11 (1968), 115-124.

[4] I. Juhász, Cardinal Functions - Ten Years Later, Math. Centre Tracts 123, Amsterdam, 1980.

[5] —, Cardinal functions II, in: Handbook of Set-Theoretic Topology, K. Kunen and J. E. Vaughan (eds.), North-Holland, Amsterdam, 1984, 63-109.

[6] I. Juhász, L. Soukup, and Z. Szentmiklóssy, Combinatorial principles from adding Cohen reals, in: Logic Colloquium '95 (Haifa), Lecture Notes in Logic 11, Springer, Berlin, 1998, 79-103.

[7] B. E. Shapirovskiı̌, Cardinal invariants in bicompacta, in: Seminar on General Topology, Moscow Univ. P.H., Moscow, 1981, 162-187 (in Russian).

[8] Z. Szentmiklóssy, S-spaces and L-spaces under Martin's axiom, in: Topology, Vol. II (Budapest, 1978), Colloq. Math. Soc. János Bolyai 23, North-Holland, Amsterdam, 1980, 1139-1145.

[9] V. V. Tkachuk, Point-countable $\pi$-bases in first countable and similar spaces, Fund. Math. 186 (2005), 55-69.

[10] S. Todorčević, Partition Problems in Topology, Contemp. Math. 84, Amer. Math. Soc., 1989.

Alfréd Rényi Institute of Mathematics

V. Reáltanoda utca, 13-15

H-1053 Budapest, Hungary

E-mail: juhasz@renyi.hu soukup@renyi.hu
Department of Analysis Eötvös Loránd University Pázmány Péter sétány 1/A H-1117 Budapest, Hungary E-mail: zoli@renyi.hu

Received 22 March 2007;

in revised form 12 May 2007 